\title{
Seminare / Séminaires / Seminari 2013
}

\section{Praxiseröffnung/-übernahme}

Das Seminar richtet sich an Ärztinnen und Ärzte, welche vor einer Praxiseröffnung (Einzel-/Gruppenpraxis), dem Einstieg in eine Gruppenpraxis oder vor einer Praxisübernahme stehen.

\section{Themen}

- Juristische Aspekte (Praxisbewilligung, Zulassung zur Sozialversicherung, Vertragswesen)

- Gesellschaftsformen/Ehe- und Erbrecht (Privat-/Geschäftsvermögen, Güterstand, Erbschaftsplanung)

- Praxiseinrichtung (Inneneinrichtung, Kostenberechnung)

- Praxisadministration (Leistungserfassungs- und Abrechnungssysteme)

- Bewertung einer Arztpraxis (Berechnung Inventarwert und Goodwill als Verhandlungsbasis)

- Finanzierung der Arztpraxis (Businessplan, Kredite, Absicherungsmöglichkeiten)

- Versicherungen/Vorsorge/Vermögen (Personen- und Sachversicherungen, Vorsorgeplanung).

\section{Sponsoren}

Die Kosten werden durch diverse Sponsoren (siehe www.fmhservices.ch) gedeckt.

\section{Daten}

K01 Donnerstag, 7. März 2013 Zürich 09.00-16.30 Uhr Volkshaus

K02 Donnerstag, 25. April 2013 St. Gallen 16.00-20.30 Uhr Hotel Einstein

K03 Donnerstag, 13. Juni 2013 Bern 9.00-16.30 Uhr Schmiedstube

\section{Praxisuibergabe}

Das Seminar richtet sich an zukünftige Praxisübergeber/innen. Idealtermin: 5-10 Jahre vor geplanter Übergabe (aus steuer- und vorsorgeplanerischen Gründen).

\section{Themen}

- Juristische Aspekte (Praxisübergabevertrag, allg. Vertragswesen, Übergabe der Krankengeschichten)

- Nachfolgeplanung und Bewertung einer Arztpraxis (projektorientiertes Vorgehen in der Nachfolgeplanung, Berechnung Inventarwert und Goodwill als Verhandlungsbasis

- Versicherungen/Vorsorge/Vermögen (Übergabe/Auflösung von Versicherungsverträgen, Pensions- und Finanzplanung)

- Steuern (Steueraspekte bei der Praxisübergabe: Optimierung der steuerlichen Auswirkungen, Liquidations- und Grundstückgewinnsteuer, Bestimmung des optimalen Übergabezeitpunktes).

\section{Sponsoren}

Die Kosten werden durch diverse Sponsoren (siehe www.fmhservices.ch) gedeckt.

\section{Daten}

K06 Donnerstag, 14. März 2013 Zürich 13.30-18.00 Uhr Volkshaus

K07 Donnerstag, 16. Mai 2013 St. Gallen 16.00-20.30 Uhr Hotel Einstein

K08 Donnerstag, 20. Juni 2013 Bern 13.30-18.00 Uhr Schmiedstube

\section{Finanz- und Steuerplanung}

Das Seminar richtet sich an Praxiseröffner/innen, Praxisübernehmer/innen sowie an bereits praxistätige Ärztinnen und Ärzte.

\section{Themen}

- Finanzplanung (Businessplan, buchhalterische Massnahmen vor Praxiseröffnung/ -übernahme, Standardkontenplan, doppelte Buchhaltung, EDV-unterstützte Buchführungslösung)

- Steuern (Steueraspekte bei Eintritt in die Selbständigkeit, Steuerfallen und Steuerrisiken, optimierte Steuerplanung).

\section{Kosten}

Für FMH Services-Mitglieder kostenlos.

\section{Daten}

K11 Donnerstag, 21. März 2013 Zürich 13.30-18.00 Uhr Volkshaus

K12 Donnerstag, 19. Sept. 2013 Bern 13.30-18.00 Uhr Schmiedstube

\section{Praxiscomputerworkshop}

Der Workshop richtet sich an praxiseröffnende sowie an bereits praxistätige Ärztinnen und Ärzte.

\section{Inhalt}

- Anforderungen an ein Praxisinformationssystem (Einführung)

- Evaluationsprozess (projektorientiertes Vorgehen in der Evaluation eines Praxisinformationssystems)

- Präsentation von sechs führenden Praxisinformationssystemen (Leistungserfassung, elektronisches Abrechnen unter Einbezug der TrustCenter, Agendaführung, Statistiken, Laborgeräteeinbindung, elektronische Krankengeschichte, Finanzbuchhaltungslösungen usw.).

\section{Kosten}

Für FMH Services-Mitglieder kostenlos.

\section{Daten}

K13 Donnerstag, 28. März 2013 Zürich 13.30-18.00 Uhr Technopark
K14 Donnerstag, 27. Juni 2013 Bern 13.30-18.00 Uhr BERNEXPO

Ouverture et reprise d'un cabinet médical Le séminaire est destiné aux médecins sur le point d'ouvrir un cabinet médical (individuel ou de groupe), de joindre un cabinet de groupe ou de reprendre un cabinet existant.

\section{Contenu}

- Business plan (préparation du plan de financement et crédit d'exploitation, financement par la banque)

- Aménagement (implantation, projet et concept d'aménagement, choix du mobilier, budget)

- Estimation d'un cabinet (inventaire et goodwill)

- Administration d'un cabinet médical (dans le cabinet, par la banque)

- Assurances (toutes les assurances à l'intérieur et autour du cabinet)

- Passage du statut de salarié à celui d'indépendant

- Fiscalité.

\section{Sponsors}

Les coûts sont pris en charge par divers sponsors (voir www.fmhservices.ch).

\section{Dates}

$\begin{array}{cl}\text { K20 Jeudi } 14 \text { mars } 2013 & \text { Lausanne } \\ \text { 13.30-18.00 h } & \text { Hôtel Alpha-Palmiers } \\ \text { K21 Jeudi } 6 \text { juin } 2013 & \text { Genève } \\ 13.30-18.00 ~ h & \text { Crowne Plaza }\end{array}$

\section{Remise d'un cabinet médical}

Le séminaire s'adresse aux médecins désirant remettre un cabinet médical. Idéalement 510 ans avant la remise prévue (pour des questions de taxation et prévoyance)

\section{Contenu}

- Aspects juridiques (autour du contrat de remise/reprise)

- Estimation d'un cabinet (inventaire et goodwill)

- Assurances (prévoyance, assurances à l'intérieur et autour du cabinet)

- Conséquences fiscales d'une remise.

\section{Sponsors}

Les coûts sont pris en charge par divers sponsors (voir www.fmhservices.ch).

\section{Dates}

\begin{tabular}{|c|c|}
\hline K24 Jeudi 25 avril 2013 & Lausanne \\
\hline $17.00-21.30 \mathrm{~h}$ & World Trade Cente \\
\hline K25 Jeudi & \\
\hline 14 novembre 2013 & Genève \\
\hline $17.00-21.30 \mathrm{~h}$ & Crowne Plaza \\
\hline
\end{tabular}


Apertura e rilevamento

di uno studio medico

Il seminario è destinato ai medici in procinto

di aprire o di rilevare uno studio medico.

\section{Contenuto}

- Business Plan (preparazione del piano di finanziamento e del credito d'esercizio, prestito bancario)

- Pianificazione (insediamento, progetto e pianificazione, scelta del mobilio, budget)

- Valutazione di uno studio medico (inventario e goodwill)

- Amministrazione di uno studio medico (interna allo studio, rapporti con la banca)

- Assicurazioni (tutte le assicurazioni necessarie interne ed esterne allo studio)

- Passaggio dallo stato di dipendente a quello di indipendente

- Fiscalità.

\section{Sponsor}

Diversi sponsor si fanno carico delle spese (si rimanda al sito www.fmhservices.ch).

\section{Seminarsponsoren 2013}

Die Unterstützung durch verschiedene Sponsoren ermöglicht es den FMH Consulting Services AG, ihre Seminarreihen für FMH Services-Mitglieder teils kostenlos, teils kostengünstig anzubieten. Gerne stellen wir Ihnen diese Firmen in einem Kurzporträt vor.

\section{medics labor}

\section{professionell und persönlich}

Medics Labor AG

Chutzenstrasse 24, 3001 Bern

Tel. 03137220 02, Fax 0313714044

info[at]medics-labor.ch

www.medics-labor.ch

Medizinisches Labor und mehr

Medics Labor ist ein Schweizer Unternehmen, zu Hause in Bern, hier verwurzelt und seit vielen Jahren erfolgreich tätig im Kanton sowie weiteren Regionen.

Geschätzt als persönliches, unkompliziertes Gegenüber, überzeugt Medics Labor durch fachliches und menschliches Gespür mit zahlreichen Hilfestellungen und Dienstleistungen. Wir verstehen uns als sozialer Arbeitgeber und beschäftigen auch behinderte Personen.

Medics Labor ist ein Labor von Ärzten für Ärzte. Es gehört den Laborspezialisten und den Ärzten, die das Unternehmen gemeinsam führen.

\section{S్jొbioanalytica}

Bioanalytica AG

Maihofstrasse 95a, 6006 Luzern
Date

\begin{tabular}{|c|c|c|}
\hline K50 & $\begin{array}{l}\text { Giovedì } 18 \text { aprile } 2013 \\
\text { dalle } 14.00 \text { alle } 18.00\end{array}$ & $\begin{array}{l}\text { Chiasso } \\
\text { FMH Fiduciaria } \\
\text { Services }\end{array}$ \\
\hline K5 & $\begin{array}{l}\text { Giovedì, } 24 \text { ottobre } 2013 \\
\text { dalle } 14.00 \text { alle } 18.00\end{array}$ & $\begin{array}{l}\text { Chiasso } \\
\text { FMH Fiduciaria } \\
\text { Services }\end{array}$ \\
\hline
\end{tabular}

\section{Anmeldung und Auskunft / Inscription et information / Iscrizioni e informazioni}

www.fmhservices.ch oder FMH Consulting Services, Cornelia Fuchs, Burghöhe 1, 6208 Oberkirch, Tel. 04192500 77, Fax 0419210586.

\section{Hinweis / Remarque / Osservazioni}

Bei sämtlichen Seminaren, bei denen die Kosten teilweise oder gänzlich von Seminarsponsoren gedeckt werden, werden die Teilnehmeradressen den jeweiligen Sponsoren zur Verfügung gestellt.

Les adresses des participants aux séminaires dont les coûts sont couverts en partie ou tota-

Tel. 04142931 31, Fax 0414293130

service[at]bioanalytica.ch

www.bioanalytica.ch

\section{Engagierte Kompetenz}

Bioanalytica, 1957 in Luzern gegründet, basiert auf einer langjährigen Tradition. Stetige Innovation und ein Team qualifizierter Fachspezialisten und Labormediziner bilden das Fundament unserer Kompetenz. Qualität und Seriosität - das sind die Werte, denen wir uns verschrieben haben. Aus der Überzeugung, dass dies auch unseren Kunden wesentliche Vorteile bietet, haben wir unser Labor im Jahre 2000 akkreditieren lassen.

Schnell, wenn notwendig auch rund um die Uhr, sind wir für Sie da. Mit dem Know-how von rund 85 Mitarbeitenden, modernster Laborautomation und Informationstechnologie sind unsere Laborresultate in kürzester Zeit verfügbar. Bei Bioanalytica stehen Sie als Kunde im Mittelpunkt. Wir unterstützen Sie und Ihr Praxisteam jederzeit gerne optimal im persönlichen Kontakt und mit zahlreichen wertvollen zusätzlichen Dienstleistungen.

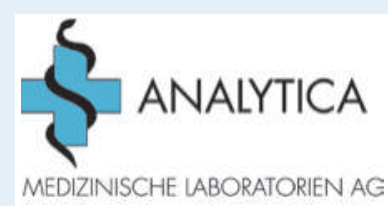

Analytica Medizinische Laboratorien AG

Falkenstrasse 14

8024 Zürich

Tel. 0442505050

kundendienst[at]analytica.ch

www.analytica.ch lement par des sponsors sont communiquées aux sponsors concernés.

Gli indirizzi dei partecipanti ai seminari, i cui costi sono coperti in parte o completamente da degli sponsor, vengono comunicati agli sponsor interessati.

\section{Annullierungsbedingungen / Conditions} d'annulation / Condizioni d'annullamento Bei Abmeldungen oder Fernbleiben werden folgende Unkostenbeiträge erhoben:

Un montant est perçu pour une absence ou une annulation. Il est de:

Un importo verrà rimborsato in caso di assenza o annullamento. Esso sarà di:

50 CHF pro Person ab 14 Tage vor Seminarbeginn / par personne dans les 15 jours avant le début du séminaire / per persona entro i 15 giorni prima dell'inizio del seminario; 100 CHF pro Person ab 7 Tage vor Seminarbeginn oder Fernbleiben / par personne dans les 7 jours avant le début du séminaire / per persona entro i 7 giorni prima dell'inizio del seminario.

Werte. Verbinden.

Sehr geehrte Frau Kollega,

sehr geehrter Herr Kollege

Die Analytica Medizinische Laboratorien AG wurde 1957 von meinem Vater gegründet und ich durfte das Labor 1985 in zweiter Generation übernehmen. Eigentlich mag ich es gar nicht, mich unpersönlich und mit schönen Worten vorzustellen. Ich bin durch und durch Praktiker, gibt es ein Problem, dann löse ich es, und kennt mich ein Kunde noch nicht persönlich, dann komme ich gerne vorbei und stelle mich vor.

Wir haben uns nach langen Diskussionen für den Slogan «Werte. Verbinden.» entschieden. Der Punkt zwischen den Worten ist kein Schreibfehler. Wir haben Werte. Werte, die uns einen persönlichen Umgang miteinander ermöglichen, Werte, die die Qualität unserer Arbeit beschreiben, und Werte, die wir mit Ihnen - unseren Kunden - teilen. Diese Werte verbinden uns miteinander und stellen das tragfähige Netz dar, das uns alle seit so vielen Jahren trägt. Für das bin ich dankbar.

Dr. med. Peter Isler

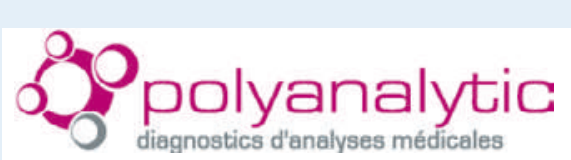

Polyanalytic SA

Avenue de Sévelin 18, 1004 Lausanne Tel. 02180492 50, Fax 0218049250 info[at]polyanalytic.ch www.polyanalytic.ch

Polyanalytic ist ein Labor für medizinische Analysen, das auf dem Gebiet der Kantone Waadt und Neuenburg tätig ist. 
Gestützt auf seine Kompetenzen, die es in den Dienst der Patientinnen und Patienten und der Ärzteschaft stellt, bietet Polyanalytic eine umfassende Palette von medizinischen Analysen.

Seit Polyanalytic 1983 gegründet wurde, ist das Unternehmen für herausragende Qualität und kundennahe Dienstleistungen bekannt. Den frei praktizierenden Ärztinnen und Ärzten werden mit unvergleichlicher Konstanz verlässliche, rasche und kompetente Leistungen geboten, damit sie ihre Kunst ausüben können.

Mit Polyanalytic verfügen die Ärztinnen und Ärzte nicht nur über einen Partner, der auf ihre Bedürfnisse eingeht, sondern auch tagtäglich über echte Unterstützung bei ihrer Tätigkeit. Polyanalytic ist mehr als ein Unternehmen: Dank der Kompetenz der Menschen, die dort arbeiten, kann die Ärzteschaft darauf vertrauen, dass bei den Patientinnen und Patienten, für die sie verantwortlich ist, optimale Laborkontrollen gewährleistet sind.

\section{S్కి dianalabs}

Dianalabs SA

Rue de la Colline 6, 1205 Genève

Tel. 02280712 40, Fax 0228071244

info[at]dianalabs.ch

www.dianalabs.ch

Dianalabs ist ein Labor für medizinische Analysen, das 1988 mit dem Ziel gegründet wurde, der Ärzteschaft und den Patientinnen und Patienten optimale Laborkontrollen zu bieten.

Aufgrund seiner Publikationen und Präsentationen ist das Genfer Labor für die Qualität seiner Serologie international anerkannt.

Wir bieten eine umfassende Palette von medizinischen Analysen, um alle Bedürfnisse der Medizin abzudecken. Doch Dianalabs ist mehr als ein polyvalentes Allround-Labor: Dank seinem Spezialistentzeam deckt es eine Vielzahl von Fachgebieten ab und bietet eine Schnittstelle zu jedem medizinischen Fachgebiet mit seinen besonderen Bedürfnissen.

Durch die wissenschaftliche Zusammenarbeit mit den Ärztinnen und Ärzten und den universitären Zentren wurde uns klar, dass nur ein regionales Unternehmen, das grundlegende menschliche Werte wie Qualität, Austausch und Dienstleistungsbereitschaft in den Vordergrund stellt und mit den lokalen Verhältnissen vertraut ist, die Bedürfnisse der Bevölkerung und der Ärzteschaft kompetent erfüllen kann.

\section{Schmid} MOGELSBERG MEDIPRINT • CLASSICPRINT

Schmid Mogelsberg AG

Sonnmattstrasse 1, 9122 Mogelsberg
Tel. 07137560 80, Fax 0713756081

info[at]schmid-mogelsberg.ch

www.schmid-mogelsberg.ch

\section{Ärztedrucksachen - 80 Jahre im Dienste der Ärzte}

Seit 80 Jahren pflegen wir die Kunst des Details. Deshalb vertrauen uns im Bereich Mediprint über 9000 Ärzte in der ganzen Schweiz. Qualität, Perfektion und Vertrauensbildung sind für uns ausschlaggebend wie für Sie als Kunde. Nehmen Sie unsere sorgfältige Beratung oder unsere Druckmusterkollektion in Anspruch. Anruf genügt.

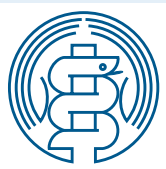

Schweizerische Ärzte-Krankenkasse Oberer Graben 37, Postfach 2046 , 9001 St. Gallen

Tel. 07122718 18, Fax 0712271828

info[at]saekk.ch

www.saekk.ch

Die richtige Adresse für Erwerbsausfalldeckungen, Kollektivkrankenkasse und Versicherungsplanung

Mit mehr als 110 Jahren Erfahrung kennt unsere Organisation auch heute die Bedürfnisse der Ärztinnen und Ärzte. Sie bietet entsprechend durchdachte und kostengünstige Lösungen an, sowohl für Praxiseröffner/innen wie auch für selbständige und angestellte Ärztinnen und Ärzte.

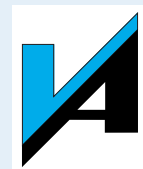

Versicherung der Schweizer Ärzte Genossenschaft Länggassstrasse 8, 3000 Bern 9

Tel. 03130125 55, Fax 0313025156

versa[at]versa.ch

www.versa.ch

Spezialisiert auf die Bedürfnisse von Ärztinnen und Ärzten und deren Ehegatten, bietet die Versicherung der Schweizer Ärzte Genossenschaft individuelle, flexible und kostenoptimierte Lösungen für Risikoabdeckungen (Tod und Invalidität) sowie für den sicheren Kapitalaufbau im Bereich der privaten Vorsorge an.

\section{kearchîv-schweiz}

kgarchiv-schweiz

Berner Archiv AG

Bernstrasse 23, 3122 Kehrsatz

Tel. 0319601070

info[at]kgarchiv.ch

www.kgarchiv.ch
Wir archivieren Krankengeschichten, Röntgenbilder und Firmenakten.

kgarchîv-schweiz bietet in Zusammenarbeit mit FMH Services ein datenschutz-konformes System, um Ärzten/-innen die Dienstleistung der Archivierung, gemäss Standesregeln, abzunehmen. Ein spezielles Angebot besteht für Arztpraxen mit unvollständiger Nachfolgeregelung.

Das Krankengeschichten-Archiv ist eine spezifische Dienstleistung der Berner Archiv AG. Die sehr sensiblen Patientendaten verlangen nach einem sorgfältigen und fachgerechten Handling, in gesicherten Archivräumen, durch entsprechend geschultes Personal. Die Dienstleistungen erfüllen die Norm ISO 9001/2008.

\section{medica}

\section{MeDIZINISCHE LABORATORIEN Dr F. KAEPPEU Aa}

\section{MEDIZINISCHE LABORATORIEN}

\section{DR. F. KAEPPELI AG}

Eidgenössisch anerkannte Laboratorien Wolfbachstrasse 17, 8024 Zürich

Tel. 044269 99 99, Fax 0442699909

info[at]medica-labor.ch

www.medica-labor.ch

Der promovierte Mikrobiologe und Biochemiker Dr. F. Käppeli, Laborspezialist FAMH, übernahm 1976 das heute über 50-jährige Unternehmen und gründete als dessen Leiter und Inhaber die Einzelfirma medica. Der wichtigste unternehmerische Leitgedanke von Dr. F. Käppeli heisst kontinuierliche Innovation und Schaffung wegweisender Standards auf allen Gebieten der Labormedizin: Mikrobiologie inklusive Parasitologie, Serologie, Immunologie, klinische Chemie, Hämatologie, molekulare Diagnostik und Pathologie in Human- und Veterinärmedizin. So entstand ein Kompetenz-Zentrum für Labordiagnostik von gesamtschweizerisch grosser Bedeutung. Die modernst ausgebauten Laboratorien werden laufend erweitert und befinden sich im Herzen von Zürich. Über 200 Angestellte der Partnerlabors, begleitet von Spezialisten aus Medizin, Pharmakologie, Naturwissenschaften und Technik, garantieren für höchste Professionalität.

\section{EGalexis}

Galexis AG

Industriestrasse 2, Postfach, 4704 Niederbipp

Tel. 05885171 11, Fax 0588517114

info[at]galexis.com

www.galexis.com

Als Vollgrossist setzt Galexis AG Standards im Schweizer Gesundheitsmarkt. Wir beliefern unsere Kunden ganz nach dem Motto «Alles aus einer Hand» mit Pharma, Praxis- und Laborbe- 
darf sowie Medizintechnik und erbringen darüber hinaus integrierte Dienstleistungen in der Gesundheitslogistik -schweizweit. Mit erprobten Lösungen fördert Galexis den Erfolg ihrer Kunden. Möchten Sie ausserdem Ihre eigene Praxis praktisch, funktionell und ästhetisch einrichten? Genau hier kann Sie Galexis mit ihren Fachpartnern und einer langjährigen Erfahrung professionell beraten und unterstützen!

Überzeugen Sie sich - mit Galexis können Sie rechnen!

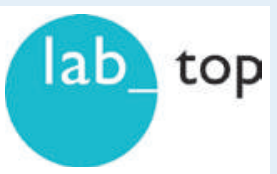

LabTop Medizinische Laboratorien AG Chriesbaumstrasse 6, 8604 Volketswil Tel. 04339930 30, Fax 0433993031 info[at]labtop.ch www.labtop.ch

\section{Das externe Labor in Ärztehand}

Ärzte halten zusammen: LabTop ist ein von Ärzten aufgebautes und von Labor-Profis geführtes Privatlabor. Seit 10 Jahren unterstützt es den Arzt in seinem Sinne. LabTop ist überwiegend im Besitz von Ärzten und steht beteiligungswilligen Ärzten weiterhin offen.

Zur Selbsthilfe von praktizierenden Ärzten gestartet, klein, modern, unabhängig, exakt - typisch schweizerisch eben - bietet LabTop bestechend einfache und modernste Lösungen für die Arztpraxis. Bei LabTop bleibt die externe Analytik in Ärztehand.

Vorteile für Sie: Als Novum bietet LabTop ein web-basiertes Geschäftsmodell, von dem Sie in verschiedener Hinsicht profitieren: RessourcenEinsparungen in Ihrer Praxis dank optimierter Prozesse, top Service, Messqualität, die höchsten Ansprüchen genügt, und nicht zuletzt, Ihr Beitrag bei der Analyseerfassung ist LabTop etwas wert.

LabTop - von Ärzten aufgebaut, für Ärzte geführt.

\section{zur Rose}

Zur Rose Suisse AG

Walzmühlestrasse 60, 8500 Frauenfeld

Tel. Ärztegrossist 0527240020

Tel. Versandapotheke 0848842842

info[at]zur-rose.ch

www.zur-rose.ch

Zur Rose - zuverlässiger und vertrauensvoller Partner von mehr als 3000 Ärztinnen und Ärzten Zur Rose ist Marktführer in der Schweizer Medikamentendistribution in den Bereichen Medikamentenversand und Arztpraxisbelieferung. Als standeseigenes Unternehmen vertritt Zur Rose die Interessen der Ärzteschaft.

\section{Zur Rose für Ärzte}

Mehr als 3000 Arztpraxen in der ganzen Schweiz erhalten von Zur Rose alles geliefert, was sie täglich benötigen: Medikamente, Impfstoffe, Insuline, Seren, Magistralitäten, Verbrauchsmaterialien, Labor- und Röntgenbedarf sowie Praxisgeräte und Instrumente aller Art.

\section{Zur Rose für Patienten}

Im Auftrag der Ärztinnen und Ärzte verschickt die Versandapotheke Zur Rose portofrei Medikamente an über 200000 Kundinnen und Kunden. Zur Rose führt die Medikamenten- oder Bezugschecks kostenfrei aus und gewährt zusätzlich bis zu 12\% Rabatt.

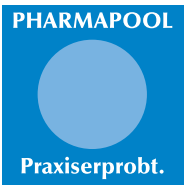

Pharmapool AG

Unterlettenstrasse 18, 9443 Widnau

Tel. 07172725 25, Fax 0717272555

info[at]pharmapool.ch

www.pharmapool.ch

Pharmapool ist der ärzteeigene Grossist mit 24-Stunden-Lieferbereitschaft. Das Kennen der Bedürfnisse von Arzt und Praxispersonal und das Wissen über die medizinischen Abläufe stehen im Mittelpunkt.

Dank der fundierten Betreuung und rationellen Belieferung wird die Wirtschaftlichkeit der von Pharmapool bedienten Praxen verbessert - sowohl von rezeptierenden als auch selbstdispensierenden Ärzten. Unabhängig von Einzelinteressen der Pharma-Branche erhalten unsere Kunden das gesamte Sortiment an Originalprodukten, Generika, Seren, Verbrauchsmaterialien, Einrichtungsgegenständen und MiGeL-Artikeln zu transparenten Konditionen. Eng verbunden mit der attraktiven Lieferpolitik sind unsere praxiserprobten Dienstleistungen rund um die Medikamenten-Logistik, wie z. B. modernes Bestellwesen sowie praktische Preisnachführung und hilfreiche Einkaufs-Statistiken. Pharmapool von Ärzten für Ärzte.

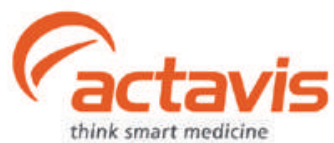

Actavis Switzerland AG

Wehntalerstrasse 190, 8105 Regensdorf-Zürich Tel. 04487097 00, Fax 0448709701

info[at]actavis.ch

www.actavis.ch
Actavis - für alle, die mehr wollen!

1956 sind in ganz Europa die Originalpräparate durch Patente geschützt, ausser in Island! Die Nachfahren der Wikinger nutzten dies und ihre Tatkraft zur Gründung von Actavis und zum Aufstieg zur weltweiten Nr. 3 unter den Generikafirmen.

Was können Sie von uns seit 2007 in der Schweiz erwarten? Erleben Sie die sprichwörtliche Verlässlichkeit, Unkompliziertheit, die Suche nach individuellen Lösungen und einen echten Mehrwert für Ihren Praxisalltag.

Wenn Sie mehr wollen als nur Medikamente, z.B. ein einzigartiges Angebot an Workshops erleben, dann sind Sie bei uns richtig. Wir wollen, dass Sie ein gutes Gefühl haben. Machen Sie Ihre persönlichen Begegnungen mit den Mitarbeitenden von Actavis - wir bemühen uns ganz besonders um Sie!

\section{Vitodata}

Vitodata AG

Deisrütistrasse 10, 8472 Oberohringen

Tel. 05232055 55, Fax 0523205566

empfang[at]vitodata.ch

www.vitodata.ch

\section{Vitodata - für Ärztinnen und Ärzte} der Zukunft

Als unabhängiges Schweizer Familienunternehmen agiert die auf 117 Mitarbeitende herangewachsene Vitodata seit über 30 Jahren im Gesundheitswesen mit dem Ziel, IT-Lösungen für praktizierende Ärzte und Ärztinnen zu entwickeln. Mit unserer neuen Applikation vitodata ist ein innovatives Praxisinformationssystem mit einem grossen Funktionsumfang verfügbar. Es setzt neue Massstäbe in Design und Benutzbarkeit und ist perfekt auf die Bedürfnisse des modernen Praxisalltags zugeschnitten. Was die Wartung, Sicherheit und den Support Ihrer IT-Infrastruktur angeht, bietet Vitodata zudem erstklassige Serviceleistungen an. Von der Software und deren sicheren Betrieb bis hin zur Hardware können Sie alles über uns beziehen. Wir unterhalten starke Partnerschaften zu Microsoft, Wortmann, Dell, Avira und Sonicwall und arbeiten dadurch stets mit den neusten Technologien und Geräten. 